\title{
Réticences françaises à la phobie de l'idylle ? - De la réception de deux romans pour adolescents suédois en France au début des années 2000
}

Valérie Alfvén

Université de Stockholm

Dans la première décennie des années deux-mille, deux romans pour adolescents Faire le mort (FM) de Stefan Casta et Quand les trains passent (QLTP) de Malin Lindroth ${ }^{\mathrm{I}}$ sont traduits du suédois et publiés en France. Par le côté sombre de leur sujet, la violence gratuite ${ }^{2}$ entre adolescents, ces deux livres suédois s'inscrivent dans la lignée d'un débat provoqué en novembre 2007 par un article du journal Le Monde (Faure, 2007). Ce dernier incrimine la littérature pour adolescents d'être trop « noire » parce qu'elle propose, selon l'auteure, des « univers sombres, voire malsains » et vise notamment QLTP. Quant à FM, il interroge et provoque selon les critiques « un malaise. Un gros. » (Citrouille, 2004 : 30-3I). Malgré tout, l'ensemble des critiques reconnaissent le caractère littéraire du roman ${ }^{3}$. Au sein de la polémique, la littérature suédoise pour adolescents fait figure d'élève indisciplinée, allant à contre-sens d'un habitus ${ }^{4}$ implicite en littérature de jeunesse et d'un paradoxe évoqué par Marc Soriano qui veut qu'une « loi nonécrite " veut que l'on évite de parler de certains sujets, mais que ce sont justement ces derniers qui intéressent les adolescents (Soriano, I 974 : I 20). Ces deux romans réalistes ${ }^{5}$ diffèrent dans leur forme mais ont pour point commun de mettre en scène des adolescents issus d'une classe dite moyenne, évoluant dans des familles plus ou moins aisées et qui, sans raisons particulières, dérapent et plongent dans une violence non provoquée. Cette violence gratuite se tourne vers un ou une adolescent(e) de leur groupe. Dans le roman FM de Casta, Kim est violemment battu par ses camarades et laissé pour mort au milieu de la forêt. Dans le livre de Lindroth, QLTP, la jeune Suzy Petterson est la victime

How to cite this book chapter:

Alfvén, V. 20I 5 . Réticences françaises à la phobie de l'idylle ? - De la réception de deux romans pour adolescents suédois en France au début des années 2000. In: Cedergren, M. et Briens, S. (eds.) Médiations interculturelles entre la France et la Suède. Trajectoires et circulations de I945 à nos jours. Pp. 248-26I. Stockholm: Stockholm University Press. DOI: http://dx.doi.org/Io.I6993/bad.s. License: CC-BY 
d'une mauvaise plaisanterie de la part de plusieurs garçons de sa classe, plaisanterie qui se finit en viol collectif.

À travers la réception de ces deux romans, cet article propose de mettre en lumière une réticence française à ce que la chercheuse suédoise Sonja Svensson appelle la phobie de l'idylle (idyllfobi), nous y revenons plus loin. En quoi ces deux romans semblent-ils provocants ? Quelles conséquences textuelles mais aussi métatextuelles découlent de cette réception ? L'origine suédoise de ces textes n'est-elle pas en soi sujet à une réticence ? FM de Stefan Casta et QLTP de Malin Lindroth ont en effet une réception française problématique et si leurs qualités littéraires sont largement reconnues, le sujet traité, celui de la violence gratuite entre adolescents, dérange au point de mettre en marche tout un système de " censure ». Plus largement, cet exemple permet alors de s'interroger sur la position de la littérature suédoise pour adolescents au sein du champ culturel et littéraire français. En effet, la réception d'une œuvre dans un système donné positionne l'œuvre dans la hiérarchie de ce système dans un rapport centre-périphérie. Ce positionnement crée une dynamique au sein du système qui porte à différentes conséquences, notamment sur la liberté prise par les traducteurs ${ }^{6}$.

\section{Accueil de la critique}

À la parution de ces deux romans, la critique s'élève en général contre la thématique choisie, celle de la violence gratuite, sujet sensible et dérangeant dans un contexte de littérature de jeunesse. Les bibliothécaires s'interrogent sur FM qui est décrit comme un « [r]oman hivernal, noir violent, trop pour certains, néanmoins profondément humain » (Citrouille, 2010). La violence décrite dans ces deux romans interpelle et rappelle que même si éditeurs et autres prescripteurs ${ }^{7}$ affirment haut et fort que l'on peut aujourd'hui parler de tout en littérature de jeunesse $^{8}$, la violence, et plus particulièrement la violence gratuite, n'est pas un sujet qui va de soi dans les romans pour adolescents. Cette dernière s'y fait malgré tout de plus en plus présente depuis le dernier quart du vingtième siècle (Delbrassine, $2006: 337$ et Ferrier, 2009 : 347) mais peu de romans osent prendre leur distance par rapport à l'habitus qui régit en littérature de jeunesse. Les anglo-saxons, par tradition, sont plus enclins à utiliser la veine réaliste pour évoquer des sujets sensibles (Melvin Burgess est un des exemples les plus connus). Les auteurs français, issus d'une autre tradition, sont plus sensibles à l'horizon d'attente des prescripteurs et à leur habitus. En cherchant des équivalents fran- 
çais de style réaliste, destinés aux adolescents de treize ans et plus et publiés dans la période 2000-2009, seul le roman de Guillaume Guéraud, Je mourrai pas gibier, est comparable dans le fait qu'il aborde une violence gratuite provoquée par un adolescent ${ }^{9}$. Roman qui, comme FM et QLTP, obtient une reconnaissance littéraire ${ }^{10}$ mais une réception mouvementée. Une critique de FM de Casta remarque également que la violence entre adolescents est "souvent passée sous silence ${ }^{\text {II }}$ et met en exergue la difficulté du traitement de la violence en littérature de jeunesse surtout si celle-ci est mêlée à un réalisme narratif extrême. Pour certains, il est même évident que le livre de Lindroth franchit la limite du livre adolescent et devrait être un livre publié côté adulte. Sur le blog des Librairies des Sorcières, on peut ainsi lire : "Quand les trains passent n'est pas à mon sens un livre pour adolescents ", soulignant alors la difficulté à poser une frontière claire entre un livre pour adolescents, jeunes adultes et adultes ${ }^{\mathrm{I2}}$.

Nous l'avons évoqué en introduction, FM et QLTP sont à contre-courant d'une loi non-écrite et implicite relevée par Soriano (I974) et que reprend le chercheur Bertrand Ferrier sur le mode de l'humour : "Les livres pour la jeunesse sont devenus une maladie mentale dont le diagnostic général est clair : pruderie de rigueur, surtout dans les prétendues audaces. »(Ferrier, 2009). Il est certain que ce jugement ne peut s'appliquer aux romans suédois traduits en français, l'accueil de la critique (entre-autres) le prouve. Il semble alors que les romans suédois traitent la violence gratuite par une écriture plus approfondie qui choque ou provoque les critiques. Mais avant même d'avoir ouvert le livre, il est possible de se demander si QLTP et FM ne souffrent pas d'une réputation qui précède la littérature de jeunesse suédoise.

\section{Réputation sulfureuse}

La Suède et sa littérature de jeunesse sont généralement connues pour leur ouverture d'esprit et leur liberté d'écriture ( $L$ a revue des livres pour enfants, février 20I I). Ainsi, il n'est pas étonnant que ce soit l'immense effigie de Fifi Brindacier (et non pas celles de Stieg Larsson, de Per Olof Enqvist ou même de Selma Lagerlöf) qui accueille les visiteurs sur le stand de la Suède, invitée d'honneur du salon du livre de Genève en 20I0. Malgré tout, l'idylle du monde d'Astrid Lindgren ne prédomine pas en France comme cela est le cas chez sa voisine allemande où la popularité d'Astrid Lindgren a joué un si grand rôle que 
l'idylle suédoise de Barn i Bullerbyn ou de Fifi Brindacier est le modèle de représentation de la littérature de jeunesse suédoise (Kärrholm \& Tenngart, 20I2). Avant même d'avoir atteint leurs lecteurs français, FM et QLTP sont marqués par la réputation de la littérature de jeunesse suédoise. Dans les années I970, les romans pour adolescents suédois influencent le champ littéraire de la jeunesse en proposant de nouveaux sujets dits sensibles comme le divorce avec Ce jeudi d'octobre d'Anna-Greta Winberg (I976) ou encore en évoquant une sexualité plus libre et la question de l'avortement avec le roman Déchirer le silence de Gunnel Beckman (1976). Les romans suédois participent largement à la mutation du champ de la littérature de jeunesse de cette époque qui voit émerger son public adolescent par la création de collections qui lui sont spécialement destinées et par l'apparition de sujets qui touchent leur public (Delbrassine, 2006 : 6I-67). Cette littérature par la qualité subversive qui lui est attribuée est souvent précédée d'une peur française comme l'exprime Anna Svenbro dans un dossier spécial à propos de la littérature nordique : "Avant de parler de fascination, il faut d'abord mettre l'accent sur la peur qui l'a souvent précédée, du moins en France, où le caractère frondeur et subversif des héros, la cruauté et la crudité des thèmes abordés a longtemps détonné dans l'univers de la littérature française pour la jeunesse qui paraît bien policée en comparaison. » (Svenbro, 20I I : 87). Svenbro perçoit parfaitement la différence d'écriture sur des thématiques sensibles et l'impact que cette écriture peut avoir en positif ou négatif. Cette réputation sulfureuse est-elle justifiée et creuse-t-elle un écart déjà préexistant avec l'horizon d'attente français?

\section{Deux romans s'écartant d'un horizon d'attente}

Si la littérature de jeunesse suédoise apparait si sombre depuis de longues années, la chercheuse Sonja Svensson, ancienne directrice du centre suédois international du livre pour enfants (Barnboksintitutet), remarque que ces sujets lourds sont historiquement largement abordés dans la littérature suédoise pour adolescents mais selon elle, il semble y avoir une explosion de ces thématiques dans les années I990, où peu de romans réalistes postmodernes suédois ont une fin heureuse. Elle s'interroge alors sur la mise en valeur des côtés sombres des adultes et des adolescents. Pour caractériser ces romans pour adolescents des années 90 en Suède, elle emploie le terme d'idyllfobi (Svensson, I995: I 8-26) que nous traduisons par phobie de l'idylle. 
En quoi consiste cette phobie ? Selon Svensson, les écrivains suédois des années 90 se font une spécialité d'explorer ce qu'il y a de plus noir et de plus lourd dans les sujets et n'hésitent pas à aborder des sujets sensibles (la drogue, la sexualité, la violence, le mal, la mort, etc.) dans un milieu hyperréaliste. Les romans de Peter Pohl sont des titres phares de cette période, comme par exemple Vi kallar honom Anna ("On l'appelle Anna ", 1987, non-traduit en français) qui raconte une terrible histoire de persécution envers un jeune adolescent nommé Anders par ses camarades de classe et de colonie de vacances. Utilisant la forme classique en littérature de jeunesse d'un « je » narrateur, ces romans ont cependant une grande ambition esthétique et n’hésitent pas à utiliser une structure ainsi qu'une temporalité complexe. La phobie de l'idylle des auteurs suédois se fait ressentir jusqu'en Italie, où une éditrice italienne de jeunesse constate :

While looking for the answer to all my questions about why the main themes of contemporary Swedish stories changes so much in the last few years, I realised that maybe these authors simply decided to ban from their stories those sheer and joyous escapes that characterised many Lindgren's stories $^{\mathrm{I3}}$. (Battista, $2008: 34$ )

En observant l'histoire littéraire de jeunesse suédoise, cette phobie ne semble pas se limiter aux seules années I 990 puisque les sujets sombres dans un cadre réaliste ne se limitent pas à cette période, cependant dans ces années, la question de la responsabilité de l'auteur commence doucement à poindre dans certains débats, alors qu'il était inexistant lors de la décennie précédente (Svensson, I999 : I I7). Svensson ne remet pas en cause cette "noirceur » mais plutôt l'usage qui en est fait dans un but commercial: un livre "corsé " se vend mieux (Svensson, I999: I 8 ). Une phobie de l'idylle serait alors une forme de fuite de l'idylle classique, une opposition à l'idylle amoureuse, mais aussi à l'idylle familiale ainsi qu'une perte d'attachement à la patrie (c'est-à-dire le pays d'origine avec ses vallées, ses forêts et la maison natale). C'est sur ce point que la phobie de l'idylle suédoise semble rejoindre Bakhtine, qui lui parle en terme plus radical de destruction de l'idylle, et qui souligne que sans idylle, le lecteur assiste à une "déshumanisation ». Bakhtine va plus loin en parlant de «négation de tous les principes moraux (...) à une désagrégation $(. .$.$) de toutes les relations humaines d'autrefois :$ amour, famille, amitié, etc. L'homme positif du monde idyllique devient comique, pitoyable et superflu »(Bakhtine, I975:376). Le but recherché par la phobie de l'idylle n'étant sans doute pas la destruction de 
l'idylle, mais sans doute par tradition littéraire, la volonté de l'éviter. Le résultat peut sembler similaire par l'impression d'un monde plus dur et sombre, mais le but recherché n'est pas la mise en risée de cette idylle. Le concept décrit par Svensson appliqué aux romans suédois permet alors de mieux comprendre et de confirmer la peur qui précède leur parution en France, expliquant également en partie l'accueil et les réticences faits à FM et QLTP.

L'autre point intéressant et attendu de la phobie de l'idylle est la fin non-heureuse qui est un moyen d'illustrer le décalage d'habitus et d'horizon d'attente entre les deux pays. La fin heureuse a une fonction en général bien précise en littérature de jeunesse. D'ordre moral, elle tente de faire oublier la méchanceté (qui prend la forme de violence gratuite ici) qui a été commise par des personnages de l'histoire. Elle a une fonction réparatrice et salvatrice en contenant et limitant la méchanceté de certains personnages. Elle offre un contrôle et comme le relève Ferrier elle offre également une immunité à l'auteur qui se met à l'abri de la loi de I949 en ne "démoralisant» pas le lecteur (Ferrier, 20I3 : 50-5I). Par cette réalité juridique qui existe bel et bien en France où l'auteur peut se voir condamner pour "démoralisation ", selon le terme de la loi, il est difficile à la phobie de l'idylle, qui suppose en partie un "non-happy end », de trouver sa place sur le marché de la jeunesse français ${ }^{\mathrm{I}}$. Mais puisque la marge de manœuvre est limitée pour les auteurs français, c'est de l'étranger qu'un renouvellement peut arriver, avec la littérature suédoise mais aussi la littérature anglo-saxonne comme le relève Virginie Douglas: " dans le dernier quart du XXème siècle, l'exemplarité des parents, tout comme la nécessité d'un happy ending, jusqu'alors considérés comme des repères essentiels pour le jeune lecteur, a commencé à être questionnée. " (Douglas, 20I3).

Publiés dans le premier quart du XXIème siècle en France, QLTP et FM n'appliquent pas une fin heureuse artificielle mais ils n'imposent pas non plus une fin malheureuse. La violence gratuite qu'ils décrivent tous les deux est un terrible récit, dans la lignée de la tradition de la phobie de l'idylle, mais la fin est une porte laissée ouverte à la réflexion du lecteur, le propulsant à un autre statut qui est celui d'un lecteur responsable, rendu capable d'analyser et de juger par lui-même ce qu'il lit. Dans le roman de Casta, Kim s'est fait battre à la limite de la mort mais il survit et ne condamne pas ses bourreaux ; il ne les livre même pas à la police. Quant à Suzy P., dans le roman de Lindroth, elle perd son procès, les coupables ne sont pas condamnés pour le viol commis et pire même, ils continuent tranquillement leur vie en se mariant et fon- 
dant une famille. Seule la narratrice qui nous livre ce récit, par cet acte de confession transmet au lecteur ses remords, mais jamais une morale affectée. Nos deux romans suédois ouvrent-ils alors la porte à une trop grande liberté du lecteur ? Par leur postulation ouverte, c'est-à-dire qui, selon l'un des critères de Poslaniec " s'adresse à un lecteur capable de coopérer à la construction du sens de ce qu'il lit, par une relation dialogique avec le texte"(Poslaniec, I997 : I50), FM et QLTP portent donc le jeune lecteur vers un statut de lecteur actif qui a la possibilité de construire par lui-même le sens du texte. Cette postulation ouverte ne peut s'inscrire que dans un champ littéraire lui-même en état d'ouverture. Par cette innovation, ces deux romans suédois obtiennent une position plus forte dans le système, c'est-à-dire plus proche du centre que de la périphérie, tout en provoquant une résistance du système.

\section{Résistances}

Il ne nous est bien évidemment impossible de parler de censure aujourd'hui comme une censure dont on parle en temps de guerre ou dans une dictature. FM et QLTP sont des traductions et la traduction ne serait-elle pas par essence " censurante " pour reprendre le terme de Berman ? En effet, comme ce dernier le souligne : « [...] toute traduction comporte une part de transformation hypertextuelle, (...) dans la mesure où elle s'effectue à partir d'un horizon littéraire » (Berman, I985: I I 8). Par censure, nous entendons « toute pratique de traduction qui imprime à l'énoncé une restriction qu'elle soit sémantique ou qu'elle porte en tout premier lieu sur le signifiant, si petite soit-elle, ou qui fait dire au texte source ce qu'il entend ne pas dire. " (Gouanvic, 2007 : I32). Ce sont ces choix de traduction qu'il est intéressant d'observer et dans FM, il est parfois possible d'observer le choix du traducteur fait en fonction de son horizon littéraire et de son habitus ainsi que ceux de l'éditeur. Agneta Ségol, qui a traduit FM, est une traductrice rôdée en traduction pour la jeunesse alors que Jacques Robnard, qui a traduit QLTP, originaire du milieu du théâtre, n'est pas un spécialiste de la littérature de jeunesse. Dans le choix sémantique d'expressions violentes, la traductrice Agneta Ségol se fait parfois plus restrictive notamment en changeant de registre comme dans ces exemples : un des adolescents du groupe s'adresse à Kim qu'ils viennent de tabasser et de mettre à terre. Ils lui disent «Skärp dig nu för fan! » (Casta, I999 : I I 8) ce que Ségol traduit par «Allez, un petit effort ! ( Casta, 2007 : I 52) et dont une traduction possible serait «Bouge-toi un peu, putain! ». Dans la phrase 
suivante : "Stick han då !»(Casta, I999: I I 8) que Ségol traduit par "Donne-lui un coup de couteau! ", nous pourrions également la traduire par un registre plus oral comme " file-lui un coup de couteau » ou encore " plante-le ». Ce changement de registre traduit une légère atténuation des passages violents qui se fait écho par la suppression quasi systématique des jurons et se faisant un indice (parmi d'autres) de l'habitus face à un sujet sensible. Il faut bien sûr prendre en compte les problèmes de traduction courant du suédois en français où par exemple, le suédois a tendance à préférer des phrases courtes et le français écrit à vouloir être plus littéraire en préférant de plus longues phrases. Mais cela peut se faire au détriment du texte d'origine et de la violence dans notre cas. Prenons encore un exemple de légère réduction de la violence tiré de FM : " Någons spark träffar mig rakt över bröstkorgen. Jag far baklänges in i elden och känner hur jag slår ansiktet i en av stenarna. Det svartnar för ögonen. Blod sipprar in i munnen.» (Casta, I999 : I 8 ) que la traductrice traduit par : " Je reçois le pied de Quelqu'un dans la poitrine et tombe à la renverse dans le feu. Ma tête heurte une pierre. Je crois tourner de l'œil. J'ai un goût de sang dans la bouche. » (pp.I 5I-I 52) Dans ce cas, le texte français est très lisible et littéraire cependant, le verbe « heurter » est sans doute plus restrictif que «slår » qui est le verbe que nous pourrions traduire par " frapper ». Le texte suédois met en scène le narrateur qui semble se dédoubler, il «sent sa tête frapper contre une des pierres ", ce qui n'apparait pas dans le texte français. Cette capacité du narrateur à se détacher de lui-même parce que la douleur est trop forte contribue à la force de la violence. Enfin, nous pouvons également remarquer une légère restriction dans « j'ai un goût de sang dans la bouche » en choisissant de mettre le « je » comme sujet au lieu du sang comme sujet de l'action : "Blodet sipprar in i munnen » que nous pourrions traduite mot à mot : "Le sang suinte dans la bouche».

Le texte n'est pas le seul à subir une restriction, les problèmes de diffusion et de facilités à accéder à l'œuvre sont aussi d'autres aspects de la censure. À la parution de Quand les trains passent en 2007, la Commission de surveillance et de contrôle des publications destinées à l'enfance et à l'adolescence, chargée notamment d'appliquer la loi de I949 sur les publications destinées à la jeunesse, envoie à l'éditeur Thierry Magnier, dans un courrier daté du 27 novembre 2007, quelques conseils concernant ce roman. Elle suggère l'apposition d'une mention en 4ème de couverture portant sur l'âge minimal conseillé et propose même quinze ans comme première limite d'âge de lecture. On évoque 
même l'idée de réduire la typographie de gros caractères propres à la collection à de plus petits, afin de rendre le livre moins accessible aux jeunes lecteurs. Tout en reconnaissant les qualités littéraires du texte, la commission a clairement peur de "passages emprunts d'une certaine violence, de nature à générer un sentiment de malaise chez un lecteur non averti. » (Delbrassine, 2008 : Io)

Du coup, marqué du sceau de la subversion ${ }^{15}$, la diffusion est plus difficile. Nous ne sommes pas en temps de guerre mais l'appareil étatique et éditorial continue à jouer son rôle.

\section{Conclusion}

L'étude de la réception de ces deux romans suédois en France permet donc de s'interroger sur la nouveauté apportée par ces romans venus de Suède. Par leur présence, ils mettent en évidence une absence du traitement de la thématique (sensible) de la violence gratuite par les auteurs de jeunesse français et s'éloignent d'un habitus qui les rend provocants. L'héritage de la phobie de l'idylle duquel ils sont issus et l'usage " à plein » de la postulation ouverte confèrent alors à ces deux œuvres une place que nous situons alors dans une périphérie du centre plutôt que dans la périphérie du système en lui-même. Cette position rendant ces œuvres importantes par leur aspect innovant plus que par leur tirage ${ }^{\mathrm{I}}$.

Les interrogations posées par les critiques montrent bien une résistance française à la phobie de l'idylle suédoise. Cette dernière ne peut qu'inévitablement évoluer dans le champ français puisqu'elle est relayée comme nous l'avons vu, par la littérature anglo-saxonne. À travers cette réception et les réticences françaises plus ou moins grande à la phobie de l'idylle, nous pouvons finalement nous interroger plus largement sur le rôle de la littérature suédoise pour adolescents dans le champ de la littérature de jeunesse - par sa liberté d'écriture et sa postulation ouverte, elle pourrait être vue comme un marqueur ou tout du moins comme une mesure d'un degré de résistance et donc d'ouverture ou de fermeture du champ littéraire de la jeunesse français mais aussi, de par sa position en périphérie du centre, le signe d'un changement de statut de la littérature de jeunesse au sein du polysystème littéraire général.

\section{Notes}

I. Le roman de Stefan Casta, Faire le mort, parait en France en 2004 aux éditions Thierry Magnier et le livre de Malin Lindroth, Quand les trains 
passent, en 2005 aux éditions Actes sud junior. Pour des raisons de commodités, nous utilisons les abréviations suivantes FM pour Faire le mort et QLTP pour Quand les trains passent. Notons que QLTP est en Suède une pièce de théâtre et connait une diffusion sous cette forme alors qu'en France, en Suisse, Belgique et Canada, le texte parait sous la forme d'un roman court.

2. Par violence gratuite, nous entendons une violence non provoquée par un évènement particulier et qui se déclenche contre un autre sujet sans raison apparente, subitement et de manière imprévue.

3. FM obtient le prix August en Suède (équivalent suédois du Prix Goncourt en France) et le prix belge Farniente en 2006 et QLTP est nominé au prix suisse des lycéens de la TSR (Télévision suisse romande).

4. Selon Shavit (I98I), le polysystème de la littérature de jeunesse est né d'un besoin du polysystème éducatif et social à qui il fallait un support à l'enseignement et l'éducation des enfants ce qui expliquerait la fonctionnalité didactique intrinsèque à la littérature de jeunesse. Au sein de ce système règne un habitus, c'est-à-dire une pratique sociale qui s'applique systématiquement et spontanément, en acceptation du monde social et de ses normes, de ses hiérarchies et de ses vertus, sur le texte de jeunesse mais aussi sur les pratiques métatextuelles. Ces habitus en littérature de jeunesse peuvent alors engendrer des comportements de censure ou autocensure tant au niveau textuel que métatextuel.

5. Un roman réaliste est une fiction qui se déroule dans un environnement connu/réel sans intervention du surnaturel ou de la science-fiction. Nous mettons également à l'écart les romans policiers et les documentaires.

6. À travers cet article, nous nous appuyons sur la théorie du polysystème élaborée dans les années I970 par Itamar Even-Zohar puis appliquée à la littérature de jeunesse par Zohar Shavit (I98I). Cette théorie décrit comment les différents systèmes sont stratifiés et mis en relation les uns avec les autres. Plus une œuvre est proche du centre du système plus elle est canonisée et son statut s'en trouve valorisé ; inversement si elle se rapproche de la périphérie du système. Ce positionnement d'une œuvre est également valable entres les systèmes, ainsi, le positionnement de la littérature de jeunesse au sein du système de la littérature générale définit son statut plus ou moins grand. Cette dynamique au sein des systèmes influence également celui de la traduction. Plus la littérature de jeunesse est proche du centre, moins l'habitus en littérature de jeunesse se fera sentir et plus particulièrement sur moins de libertés seront prises dans les traductions.

7. Les prescripteurs sont, en littérature de jeunesse, tous les intervenants qui prescrivent le livre avant qu'il ne parvienne à son lecteur. Il peut s'agir d'institutions comme l'éducation nationale et le corps enseignant, comme les bibliothécaires mais aussi les éditeurs, les libraires sans oublier les parents. Cf. 
le schéma du système de transmission d'un livre de l'auteur vers son lecteur, décrit par Peter Hunt (I99I : I 58 )

8. Source : Table ronde au Salon du livre de Montreuil à Paris en novembre 2009 avec notamment les représentants des éditeurs Thierry Magnier et Hachette.

9. Il y a un vacuum dans le polysystème de la littérature de jeunesse français au début des années deux-mille. Peu ou pas d'auteurs français choisissent d'écrire pour des adolescents (de treize ans et plus) sur des adolescents violents en mêlant ce sujet à une écriture hyperréaliste. En auteur français, nous n'avons pu recenser que Guillaume Guéraud et son court roman réaliste Je mourrai pas gibier qui reçoit le prix des Sorcières en 2007 , ainsi que le roman épistolaire de Sarah K. Connexions dangereuses (2002) et Adieu la chair (2007) de Julia Kino. En sortant des frontières du roman, en bande-dessinée, la série Seuls, parue aux éditions belges Dupuis dont le premier des huit tomes parait en 2005 et le dernier en 20I3, retrace l'histoire de cinq enfants qui se retrouvent seuls dans une grande ville et qui doivent se débrouiller sans l'aide des adultes qui ont disparu mystérieusement.

Io. Le roman de Guillaume Guéraud a été distingué dans la catégorie « Romans adolescents du prix des sorcières 2007 ». Le livre a donné suite à des mises en scène (Boulogne sur mer, 2009) puis à une version en bande-dessinée en 2009 publiée chez Delcourt et qui reçoit en 20I I le " prix littéraire des lycéens et apprentis de la région Provence Alpes Côte d'Azur ».

I I. Blog de la bibliothèque de Saint-Quentin-en-Yvelines: "un livre ado "coup de poing " (sans mauvais jeu de mots...) sur les violences collectives chez les jeunes, souvent passées sous silence... " (nous soulignons).

I 2. Notons ici la difficulté à définir le public ciblé par le texte (s'il y en a un à l'origine ?). Le texte suédois est à l'origine une pièce de théâtre jouée pour un public adulte et adolescent. En France, la pièce est d'abord traduite pour une mise en scène en 2007 par la finlandaise Tiina Kaartama, qui vise un public adulte. La pièce est ensuite publiée par Actes Sud junior sous forme de court roman avec suppression des didascalies et qui cette fois vise un public adolescent. Autre trace de la difficulté à classer ce texte, les catalogues des bibliothèques municipales de Genève et Berne en Suisse le classent tantôt en section adulte, tantôt en section adolescent.

I3. Notre traduction: «En cherchant une réponse à toutes mes questions sur la raison pour laquelle les principaux thèmes des histoires suédoises contemporaines ont tellement changé au cours des dernières années, j'ai réalisé que peut-être ces auteurs ont simplement décidé de bannir de leurs histoires ces évasions abruptes et joyeuses qui caractérisent de nombreuses histoires de Lindgren. » 
I4. Notons ici que la norme juridique de la reconstruction d'après-guerre est celle qui est encore en vigueur aujourd'hui.

I 5. Le terme de "subversion » peut paraître fort, mais il reprend ici le titre utilisé pour l'émission radiophonique "La grande table », France Culture, du 2012/05/I I : "La subversion dans la littérature pour la jeunesse " et qui sous-entend que des tabous existent en littérature de jeunesse. http://www. franceculture.fr/emission-la-grande-table-ı ere-partie-la-subversion-dans-lalitterature-pour-la-jeunesse-20 I 2-05-I I. (dernière lecture 20I 5/O2/I 8)

I6. Depuis sa parution FM s'est vendu à environ 6586 exemplaires (les deux éditions confondues), nous n'avons pas pu obtenir les chiffres exacts des ventes de QLTP en France, mais après un entretien avec l'éditeur, nous avons su qu'un tirage moyen est de l'ordre de 3 ०००-3500 exemplaires. QLTP n'a pas encore été réédité.

\section{Bibliographie}

\section{Fuvres étudiées}

Casta, S. (I999), Spelar död. Opal bokförlag.

- (2004), Faire le mort. Éditions Thierry Magnier.

Lindroth, M. (2005), När tågen går förbi. Dramaten förlag.

- (2007), Quand les trains passent. Actes sud junior.

\section{Ouvrages cités}

Bakthine, M. (I978), Esthétique et théorie du roman. Paris : Gallimard.

Delbrassine, D. (2006), Le roman pour adolescents aujourd'hui : écriture, thématiques et réception. Créteil : SCÉRÉN-CRDP \& La joie par les livres.

Ferrier, B. (2009), Tout n'est pas littérature ! La littérarité à l'épreuve des romans pour la jeunesse. Collection Interférences : Presses Universitaires de Rennes.

Gouanvic, J.-M. (2007), Pratique sociale de la traduction : le roman réaliste américain dans le champ littéraire français (I920-1960). Arras : Artois Presses Université.

Hunt, P. (I99I), Criticism, Theory, and Children's Literature. Basil Blackwell.

Kärrholm, S. \& Tenngart, P. (2012), Barnlitteraturens värden och värderingar. Lund : Studentlitteratur. 
Poslaniec, C. (1999), L'évolution de la littérature de jeunesse, de I 850 à nos jours an travers de l'instance narrative. Villeneuve-d'Ascq : Presses universitaires du septentrion.

Soriano, M. (1974), Guide de la littérature pour la jeunesse. Courants, problèmes, choix d'auteur. Paris : Flammarion.

\section{Articles}

(novembre 2004), "Romans ados, Sélection Librairies des sorcières ". Citrouille $39: 30-3 \mathrm{I}$.

Battista, A. (2008), "The lost joys of a carefree life or are contemporary Swedish authors " banning " happiness ? ", Barnboken $2: 30-36$.

Delbrassine, D. (2008), "Censure et autocensure dans les romans pour la jeunesse ». Paroles $2: 8-\mathrm{I} 2$.

Douglas, V. (décembre 2013), "Quand les parents deviennent des méchant dans la fantasy britannique pour la jeunesse : le cas de Coraline de Neil Gaiman et de Luka et le feu de la vie de Salman Rushdie ». Les cabiers du CRILJ 5 .

Even-Zohar, I. (I990), "The Position of Translated Literature within the Literary Polysystem ». Poetics Today (vol.II) I : 45-5 I.

Faure, M. (30/II/2007), " Un âge vraiment pas tendre, mal-être, suicide, maladie, viol... Pourquoi les livres destinés aux adolescents sont-ils si noirs ? ", Le Monde.

Ferrier, B. (décembre 2013), "Les méchants, des personnages comme il en faut ». Cahiers du CRILJ $5: 50-5 \mathrm{I}$.

Gégène (mars 20I0), "Ce roman a pris trois ans de ma vie ». Citrouille 55 : $45-47$.

Shavit, Z. (I98I), "Translation of Children's Literature as a Function of Its Position in the Literary Polysystem ". Poetics Today 2: 4, "Translation Theory and Intercultural Relations": I7I-I79. [En ligne] http://www.jstor. org/stable/I772495 (Dernière lecture 20I5/O2/I 8)

Svenbro, A. (20I I), "Quelques repères historiques et culturels ». La revue des livres pour enfants 257 (février). Dossier spécial "Les pays nordiques ", BNF/La joie par les livres : 83-88.

Svensson, S. (I995), « Tankar kring några tendenser i 90-talets ungdomsbok ». Abrakadabra (oktober) : I 8-26.

— (I999), «Dödspolare, skuggmän och förlorade fäder. Idyllfobin i I990-ta- 
lets ungdomsbok ", in Flatekval, E \& al. Förankring och förnyelse. Nordiska ungdomsromaner inemot àr 2000. Cappelen Akademisk Forlag/LNU : I07-I2I.

\section{Références internet}

Blog, Bibliothèque de Saint Quentin en Yvelines : http://www.biblioblog.sqy.fr/ bouquins-blog/faire-le-mort-de-stefan-castal (Dernière lecture $03 / 03 / 2015$ ) 